\title{
In Vivo Selection of Porin-Deficient Mutants of Klebsiella pneumoniae with Increased Resistance to Cefoxitin and Expanded-Spectrum Cephalosporins
}

\author{
LUIS MARTÍNEZ-MARTÍNEZ ${ }^{1 *}$ SANTIAGO HERNÁNDEZ-ALLÉS, ${ }^{2}$ SEBASTIÁN ALBERTÍ,${ }^{2} \dagger$ \\ JUAN M. TOMÁS, ${ }^{3}$ VICENTE J. BENEDI, ${ }^{2}$ AND GEORGE A. JACOBY ${ }^{1} \ddagger$ \\ Infectious Disease Unit, Massachusetts General Hospital, Boston, Massachusetts, ${ }^{1}$ and Area de Microbiología, \\ Departamento de Biología Ambiental, Universidad de las Islas Baleares, Palma de Mallorca, ${ }^{2}$ and \\ Department of Microbiology, School of Biology, University of Barcelona, Barcelona, ${ }^{3}$ Spain
}

Received 14 June 1995/Returned for modification 14 September 1995/Accepted 21 November 1995

\begin{abstract}
Four Klebsiella pneumoniae isolates (LB1, LB2, LB3, and LB4) with increased antimicrobial resistance were obtained from the same patient. The four isolates were indistinguishable in biotype, plasmid content, lipopolysaccharide, and DNA analysis by pulse-field gel electrophoresis. Isolate LB1 made TEM-1 and SHV-1 $\beta$-lactamases. Isolates LB2, LB3, and LB4 produced SHV-5 in addition to TEM-1 and SHV-1. MICs of cefoxitin, ceftazidime, and cefotaxime against $\mathrm{LB1}$ were 4,1 , and $0.06 \mu \mathrm{g} / \mathrm{ml}$, respectively. MICs of ceftazidime against $K$. pneumoniae $\mathrm{LB2}$, LB3, and LB4 were $>256 \mu \mathrm{g} / \mathrm{ml}$, and those of cefotaxime were 2, 4, and $64 \mu \mathrm{g} / \mathrm{ml}$, respectively. MICs of cefoxitin against $K$. pneumoniae LB2 and LB3 were $4 \mu \mathrm{g} / \mathrm{ml}$, but that against $K$. pneumoniae LB4 was $128 \mu \mathrm{g} / \mathrm{ml}$. K. pneumoniae $\mathrm{LB} 4$ could transfer resistance to ceftazidime and cefotaxime, but not that to cefoxitin, to Escherichia coli. Isolate LB4 and cefoxitin-resistant laboratory mutants lacked an outer membrane protein of about $35 \mathrm{kDa}$ whose molecular mass, mode of isolation, resistance to proteases, and reaction with a porin-specific antiserum suggested that it was a porin. MICs of cefoxitin and cefotaxime reverted to 4 and $2 \mu \mathrm{g} / \mathrm{ml}$, respectively, when isolate LB4 was transformed with a gene coding for the $K$. pneumoniae porin OmpK36. We conclude that the increased resistance to cefoxitin and expanded-spectrum cephalosporins of isolate LB4 was due to loss of a porin channel for antibiotic uptake.
\end{abstract}

Klebsiella pneumoniae is an important human pathogen (5, 20). Cephalothin- and gentamicin-resistant strains have been responsible for nosocomial outbreaks during the 1970s (38); outbreaks caused by strains producing extended-spectrum $\beta$-lactamases (ESBL) have been described since the 1980s (16, $27,33,37)$. In most cases, ESBL are plasmid-mediated enzymes providing resistance to oxyiminocephalosporins (cefotaxime, ceftazidime, and ceftriaxone, etc.) and to aztreonam. Less often, other enzymes (MIR-1, CMY-1, FOX-1, and LAT-1, etc.), degrading $\alpha$-methoxycephalosporins (cefoxitin and cefotetan, etc.) as well as oxyimino- $\beta$-lactams, have also been described (15). A recent study on the susceptibilities of ESBL-producing $K$. pneumoniae strains has shown that about $25 \%$ of them are resistant to cefoxitin (27), but the actual mechanism of this resistance has not been precisely defined.

Porin loss as a cause of antimicrobial resistance has been shown in both laboratory mutants and clinical isolates (29). Two major porins, OmpF and $\mathrm{OmpC}$, have been described for Escherichia coli. Although OmpC and OmpF porin homologs have been described for other members of the family Enterobacteriaceae (18), the lack of a good definition of K. pneumoniae porins has resulted in their having an inferred, rather than demonstrated, role in antimicrobial resistance in this species $(11,31,40,47)$. Besides the phosphate starvation-inducible PhoE porin (48) and the LamB maltoporin (50), two nonspecific pore proteins (porins), named $37 \mathrm{kD}$ and $39-40 \mathrm{kD}$, have

\footnotetext{
* Corresponding author. Present address: Department of Microbiology, School of Medicine, University of Seville, Apdo. 914, 41080 Seville, Spain. Phone: 34-5-4557448. Fax: 34-5-4377413.

$\dagger$ Present address: Channing Laboratory, Brigham and Women's Hospital and Harvard Medical School, Boston, MA 02115.

$\ddagger$ Present address: Lahey Clinic, Burlington, MA 01805.
}

been characterized by their molecular masses and pore sizes in Enterobacter cloacae strains (19) that were later reidentified as $K$. pneumoniae (41). More recently, we have described the existence of two $K$. pneumoniae porins called OmpK36 and OmpK35 $(1,13)$. While characterization of OmpK35 is still in progress, the OmpK36 porin, a homolog of the E. coli OmpC porin, has been characterized in greater detail, and both the ompK36 nucleotide sequence and a three-dimensional model are available (2).

While monitoring an outbreak of $K$. pneumoniae producing SHV-5 $\beta$-lactamase, we noted several isolates that were resistant to cefoxitin, which is not a good substrate for this enzyme. This study was undertaken to analyze the mechanism of resistance of four isolates, obtained from the same patient, that showed increasing resistance to $\beta$-lactams and to evaluate the role of outer membrane porin alterations.

(Part of this work was presented at the 34th Interscience Conference on Antimicrobial Agents and Chemotherapy, Orlando, Fla., 1994 [22].)

\section{MATERIALS AND METHODS}

Bacterial strains. $K$. pneumoniae LB1, LB2, LB3, and LB4 were obtained from the sputum of a patient with pneumonia at Massachusetts General Hospital (Boston, Mass.). Organisms were identified by standard biochemical methods and with Pasco panels (Difco, Detroit, Mich.) according to the manufacturer's instructions. Isolates LB1, LB2, LB3, and LB4 were obtained from the same patient. The patient had been treated previously with gentamicin, and amikacin, cefotetan, and ciprofloxacin but not with oxyimino- $\beta$-lactams.

Cefoxitin-resistant mutants derived from isolates LB1, LB2, and LB3 were obtained on gradient plates containing cefoxitin, as previously described (43). E. coli J53-2 ( $\mathrm{F}^{-}$met pro $\left.\mathrm{Rif}^{\mathrm{r}}\right)$ (6) was used as the recipient strain in conjugation experiments to study plasmid-transferable $\beta$-lactamases. E. coli DH5 $\alpha$ (Life Technologies Inc.) was used to maintain genetically engineered plasmids originated during this study. K. pneumoniae C3 (44) has been used previously for porin studies in K. pneumoniae. It was isolated from sewage. It produces both 
OmpK35 and OmpK36. K. pneumoniae 96D is a previously described clinical isolate (32) producing the AmpC-type cefoxitin-degrading $\beta$-lactamase MIR-1. Strains were stored at $-70^{\circ} \mathrm{C}$ in tryptic soy broth containing $10 \%$ glycerol.

Susceptibility testing. MICs of antimicrobial agents were determined by microdilution according to the guidelines of the National Committee for Clinical Laboratory Standards with cation-adjusted Mueller-Hinton broth (Ca-MHB) (Difco). MICs were also determined in L broth (Bacto Tryptone, $10 \mathrm{~g}$; yeast extract, $5 \mathrm{~g}$; sodium chloride, $5 \mathrm{~g}$; $\mathrm{NaOH}, 1 \mathrm{mM})$ plus glucose $(2.5 \mathrm{~g} /$ liter $)$ (LBG medium) or in nutrient broth (NB) (Difco). MICs of K. pneumoniae KT5005 and KT5007 (see below) were determined in MHB supplemented with $30 \mu \mathrm{g}$ of potassium tellurite per $\mathrm{ml}$ to maintain the plasmids they contain. For three cefoxitin-resistant mutants, LB2-FOX12, LB2-FOX32, and LB2-FOX64 (see below), the MICs of cefoxitin were determined on Mueller-Hinton agar with E-test strips (AB Biodisk, Solna, Sweden).

Typing methods. Biotypes were determined with Pasco panels (type 4G; Difco) according to the manufacturer's instructions. Pulsed-field gel electrophoresis of genomic DNAs from isolates LB1, LB2, LB3, and LB4 digested with $X b a \mathrm{I}$ was performed by F. Tenover (Centers for Disease Control) (23). Analysis of plasmid DNAs carried by isolates LB1, LB2, LB3, and LB4 was performed by $0.5 \%$ agarose gel electrophoresis (39) except that $K$. pneumoniae strains were grown in the presence of $0.5 \mathrm{mM}$ bismuth nitrate- $2.5 \mathrm{mM}$ sodium salicylate to reduce capsule synthesis (9).

Mating and curing experiments. Transfer of resistance from $K$. pneumoniae isolates LB1, LB2, LB3, and LB4 to E. coli J53-2 was obtained by mating experiments (34). Ceftazidime $(10 \mu \mathrm{g} / \mathrm{ml})$, cefoxitin $(10 \mu \mathrm{g} / \mathrm{ml})$, amikacin $(10$ $\mu \mathrm{g} / \mathrm{ml})$, or gentamicin $(10 \mu \mathrm{g} / \mathrm{ml})$ was used as the selecting agent. For curing experiments, $K$. pneumoniae LB2, LB3, and LB4 were cultured on tryptic soy broth (Difco) at $42^{\circ} \mathrm{C}$ with or without $25 \mu \mathrm{g}$ of ethidium bromide (Sigma, St. Louis, Mo.) per ml. Tubes were subcultured daily in the same medium for up to 10 days and plated on tryptic soy agar. After a 24-h incubation period, derivatives susceptible to ceftazidime or cefoxitin were selected by replica plating.

$\boldsymbol{\beta}$-Lactamase characterization. K. pneumoniae LB1, LB2, LB3, and LB4 were cultured on LBG. Cells were disrupted by sonication, and after removal of unbroken cells and cell walls by centrifugation, the supernatant fluids were analyzed for $\beta$-lactamase by isoelectric focusing (24). pIs were determined by using a set of different $\beta$-lactamases with known pIs. $\beta$-Lactamase activity was revealed with nitrocefin $(0.5 \mathrm{mg} / \mathrm{ml})$. The supernatant fluids used for isoelectrofocusing were also used to evaluate their abilities to break down cefazolin, ceftazidime, cefotaxime, aztreonam, cefoxitin, and imipenem. Antimicrobial paper disks were impregnated with supernatant fluids from the different isolates, and the inhibition zones produced by them and by nonimpregnated disks against E. coli J53-2 were compared. A reduction of the zone of the activity with the impregnated disk was considered evidence for $\beta$-lactamase activity against the corresponding antimicrobial agent.

Isolation and analysis of outer membrane components. Cell envelopes (cytoplasmic and outer membranes) were isolated from $K$. pneumoniae strains grown in LBG, NB, and Ca-MHB medium by centrifugation at $100,000 \times g$ for $1 \mathrm{~h}$ at $4^{\circ} \mathrm{C}$ after French press cell lysis. Outer membrane proteins (OMP) were isolated as sodium lauryl sarcosynate-insoluble material $(10,44)$. Isolation of the $35-\mathrm{kDa}$ OMP from strain LB1 was performed by a combination of two methods for porin isolation $(28,30)$, as described for the isolation of OmpK36 porin (1). Lipopolysaccharide (LPS) was purified by the phenol-water method (51) or obtained from proteinase K-treated cell lysates (12).

Electrophoretic analysis of OMP by sodium dodecyl sulfate-polyacrylamide gel electrophoresis (SDS-PAGE) was performed with $11 \%$ acrylamide- $0.35 \%$ bisacrylamide- $0.1 \%$ SDS by using Laemmli's buffers (21) and Coomassie blue staining. Samples were boiled for $5 \mathrm{~min}$ in sample buffer before electrophoresis. Densitometric analysis of OMP separated by SDS-PAGE was carried out with a Bio Image densitometer and Whole band 3.1 software (Millipore). LPS, either purified as described above or from proteinase K-treated cell lysates (12), was analyzed by electrophoresis in $15 \%$ polyacrylamide gels and silver staining (46). Western blot (immunoblot) analysis of SDS-PAGE-separated OMP was carried out with the buffers and conditions described by Towbin et al. (45), Immobilon $\mathrm{P}$ membranes (Millipore), rabbit antiserum raised against purified OmpK36 porin (2), and alkaline phosphatase-labeled anti-rabbit immunoglobulin G (Sigma). Enzyme was detected with BCIP-NBT (5-bromo-4-chloro-3-indolylphosphate toluidinium-nitroblue tetrazolium) (3).

Plasmid construction. Plasmids encoding the complete $K$. pneumoniae porin OmpK36 or a truncated version were constructed and tagged with a tellurite resistance cassette to allow selection of plasmid-containing clones in the multiresistant background of isolate LB4. The plasmid construction strategy is shown in Fig. 1. Plasmid pDT1558 (49) was used as source of the tellurite resistance cassette kilA-Ter , which was cloned into pUC18Not (14), yielding plasmid pSHA1. The kilA-Te ${ }^{\mathrm{r}}$ cassette was then obtained as a NotI-NotI fragment from pSHA1 and was cloned into the unique NotI site of pSUV7 (2), a pACYC184derived plasmid carrying the complete ompK36 gene. The resulting pSHA2 plasmid was introduced into K. pneumoniae LB4, giving strain KT5005. Plasmidcontaining clones were selected with potassium tellurite $(30 \mu \mathrm{g} / \mathrm{ml})$ and chloramphenicol $(25 \mu \mathrm{g} / \mathrm{ml})$. The NruI-XmnI ompK36-containing fragment of pSUV7 was isolated and used to replace the HindII-HindII fragment of plasmid pACYC184. The resulting pFR165 plasmid contains two HindII sites, located within the ompK36 gene. Elimination of the intra-ompK36 HindII-HindII frag- ment of pFR165 gave plasmid pFR167, which was afterwards labeled with tellurite resistance by cloning the kilA $-\mathrm{Te}^{\mathrm{r}}$ cassette from pSHA1 into its unique NotI site. Therefore, the resulting pSHA4 plasmid contains a truncated ompK36 gene ( $\Delta$ ompK36). Selection of tellurite-resistant LB4 clones containing plasmid pSHA4 (strain KT5007) was performed as described above. Plasmids were introduced in K. pneumoniae LB4 by transformation or electroporation.

Restriction endonucleases, T4 DNA ligase, and calf intestinal alkaline phosphatase were from Pharmacia. Large- and miniscale plasmid isolation were carried out by alkaline lysis (8). DNA procedures for agarose gel electrophoresis, plasmid transformation, and recovery of DNA fragment from gels were standard (39).

\section{RESULTS}

Typing methods. $K$. pneumoniae isolates LB2, LB3, and LB4 were indistinguishable in biotype: 777727 711. Isolate LB1 differed slightly (777 727 311) because of its cephalothin susceptibility. Analysis of plasmid and total DNAs gave identical results for all four isolates: each carried four plasmids of about 180, 120, 3.9, and $3.1 \mathrm{~kb}$ (Fig. 2A), and the same pulsed-field gel electrophoresis patterns were obtained (Fig. 2B). Identical LPS profiles were also observed for isolates LB1, LB2, LB3, and LB4 after electrophoretic and silver staining analysis of both isolated LPSs and proteinase K-treated whole cells (data not shown). Electrophoretic profiles consisted of high-molecular-weight LPS (O-antigen-enriched molecules) and low-molecular-weight LPS (core-enriched molecules).

Susceptibility testing. Table 1 shows that isolate LB1 was susceptible to both expanded-spectrum cephalosporins and cefoxitin, isolates LB2 and LB3 were resistant to expanded-spectrum cephalosporins but susceptible to cefoxitin, and isolate LB4 was resistant to both expanded-spectrum cephalosporins and cefoxitin. All isolates were susceptible to imipenem and resistant to tetracycline. K. pneumoniae LB1 was susceptible to amikacin; isolates LB2, LB3, and LB4 were not. Isolates LB1, LB2, and LB4 were resistant to gentamicin and chloramphenicol, but isolate LB3 was susceptible to both agents. Although all four isolates were susceptible to ciprofloxacin, isolate LB4 was twice less susceptible than isolates LB1, LB2, and LB3. Variations between MICs obtained in LBG and NB media were within one twofold dilution, except for aminoglycosides, whose MICs were four- to eightfold lower in NB than in CaMHB medium. MICs of cefoxitin for mutants LB2-FOX12, LB2-FOX32, and LB2-FOX64, were 12, 32, and $64 \mu \mathrm{g} / \mathrm{ml}$, respectively, as determined by the E-test. MICs of cefoxitin for mutants LB1-FOX256, LB2- FOX256, and LB3-FOX256 were $>256 \mu \mathrm{g} / \mathrm{ml}$.

Mating and curing experiments. The $120-\mathrm{kb}$ plasmid coded for resistance to expanded-spectrum cephalosporins and amikacin, and the $180-\mathrm{kb}$ plasmid coded for resistance to gentamicin and chloramphenicol, as shown by conjugation experiments. Cefoxitin resistance could not be transferred by conjugation from K. pneumoniae LB4 to E. coli J53-2. Ceftazidime-susceptible mutants were obtained from isolates LB2, LB3, and LB4. They also became susceptible to aztreonam (and to amikacin) and had increased susceptibility to cefotaxime, but plasmid analysis for these mutants still revealed the presence of the $120-\mathrm{kb}$ plasmid (data not shown); mutants derived from LB2 and LB3 were susceptible to cefoxitin, but mutants derived from LB4 still were resistant to cefoxitin (MIC, $128 \mu \mathrm{g} / \mathrm{ml}$ ). Cefoxitin-susceptible mutants could not be obtained from isolate LB4.

$\boldsymbol{\beta}$-Lactamase characterization. $K$. pneumoniae LB1 produced TEM-1 (pI, 5.4) and SHV-1 (pI, 7.6). Isolates LB2, LB3, and LB4 produced SHV-5 (pI 8.2) in addition to TEM-1 and SHV-1. Supernatants containing crude $\beta$-lactamases from LB1, LB2, LB3, and LB4 did not affect inhibition zones of $E$. coli J53-2 around cefoxitin (or imipenem) disks. The superna- 


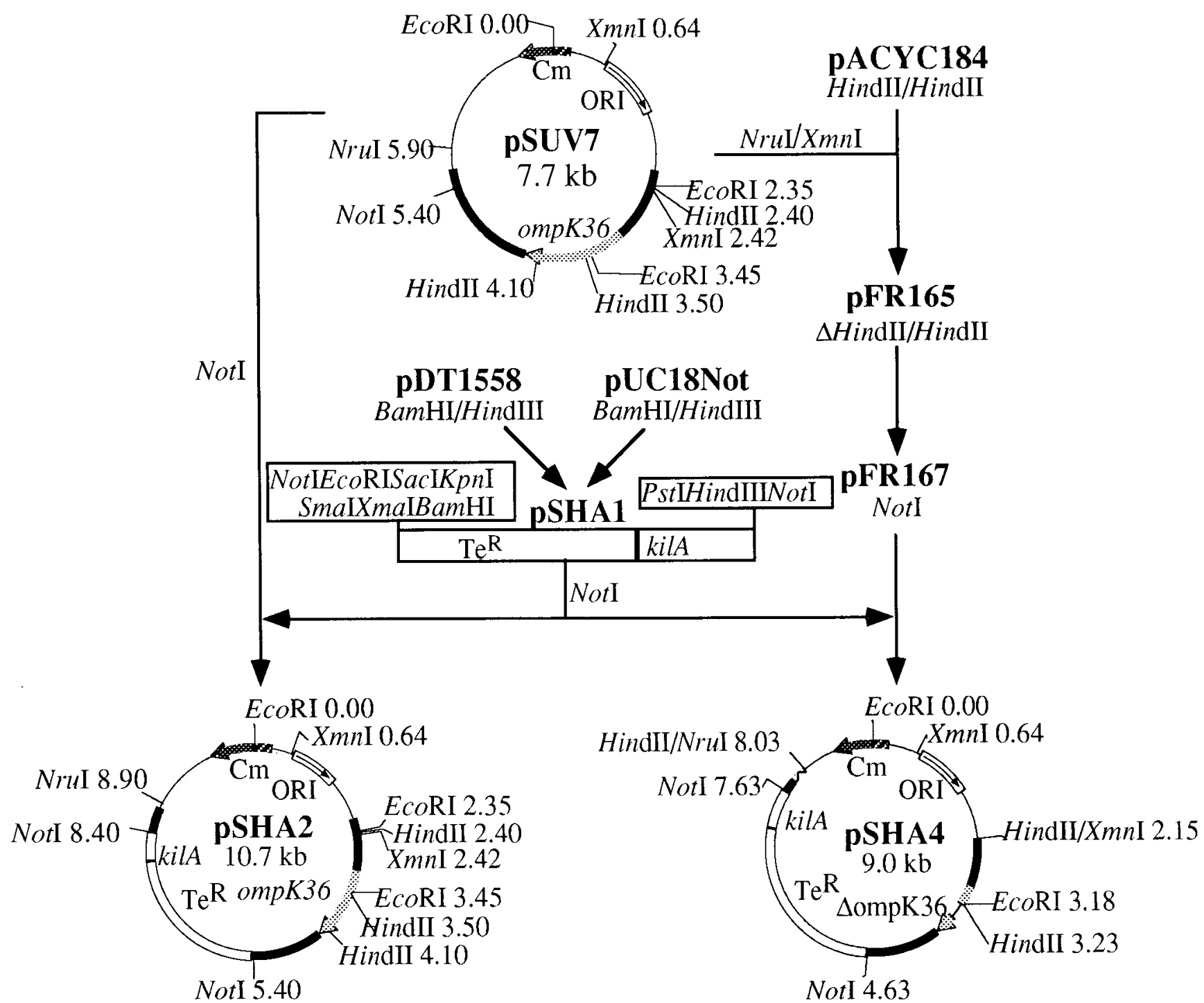

FIG. 1. Construction of plasmids containing the porin-coding ompK36 gene selectable in the multiresistant K. pneumoniae LB4 background. Plasmid pSUV7 contains the entire ompK36 gene. pSUV7 was modified by cloning the kilA-Te ${ }^{\mathrm{r}}$ cassette, which confers resistance to potassium tellurite. The resulting pSHA2 plasmid confers an OmpK $36^{+}$phenotype after introduction into K. pneumoniae LB4. As a control, plasmid pSHA4, which contains kilA-Te ${ }^{\mathrm{r}}$ for selection and a HindII-HindII intra-ompK36 deletion, was used.

tants from LB2, LB3, and LB4 antagonized the activities of disks of cefazolin, ceftazidime, cefotaxime, and aztreonam.

OMP characterization. SDS-PAGE analysis (Fig. 3) of the OMP of $K$. pneumoniae isolates grown in LBG or NB medium (high- and low-osmolarity media, respectively) showed only two major proteins, of about 32 and $35 \mathrm{kDa}$, in the 29- to $45-\mathrm{kDa}$ range for isolates $\mathrm{LB} 1, \mathrm{LB} 2$, and LB3. The $35-\mathrm{kDa}$ OMP was absent in isolate LB4 and the ceftzidime-susceptible mutants derived from it. Mutants LB2-FOX12 and LB2FOX32 also produced the 32- and 35-kDa OMP (not shown). Mutant LB2-FOX64 produced only the 32-kDa OMP (Fig. 3, lane 5). Therefore, mutants with high levels of resistance to cefoxitin, such as LB2-FOX64 (MIC, $64 \mu \mathrm{g} / \mathrm{ml}$ ) and LB1FOX256, LB2-FOX256, and LB3-FOX256 (MICs, >256 $\mu \mathrm{g} /$ $\mathrm{ml}$ ), do not express the $35-\mathrm{kDa}$ porin, whereas mutants with cefoxitin MICs of up to $32 \mu \mathrm{g} / \mathrm{ml}$ still express variable amounts of the 35-kDa OMP. Densitometric analysis of SDS-PAGEseparated OMP revealed that the ratio between the 32- and
35-kDa bands decreased by 22 to $35 \%$ in isolates LB2 and LB3 compared with isolate LB1. The same OMP electrophoretic pattern was observed when isolates were grown in the $\mathrm{Ca}$ MHB medium used for MIC determinations. As controls, $K$. pneumoniae 96D (not shown) and C3 showed the 32- and $35-\mathrm{kDa}$ OMP and an additional OMP of about $36 \mathrm{kDa}$ (the OmpK36 porin) when grown in L broth (Fig. 3).

The porin nature of the $35-\mathrm{kDa}$ OMP present in isolates LB1, LB2, and LB3 and absent from isolate LB4 (and from cefoxitin-resistant mutants derived from the first three isolates) was studied (Fig. 4). The outer membrane of LB1 was isolated and subjected to a combination of two typical methods for the isolation of porins based on their trypsin resistance and strong noncovalent association with the peptidoglycan. The peptidoglycan-associated, trypsin-resistant material of isolate LB1 was separated by gel permeation chromatography into three peaks (Fig. 4A). SDS-PAGE and Coomassie blue staining (Fig. $4 B$ ) of these chromatographic peaks revealed that peak I con- 


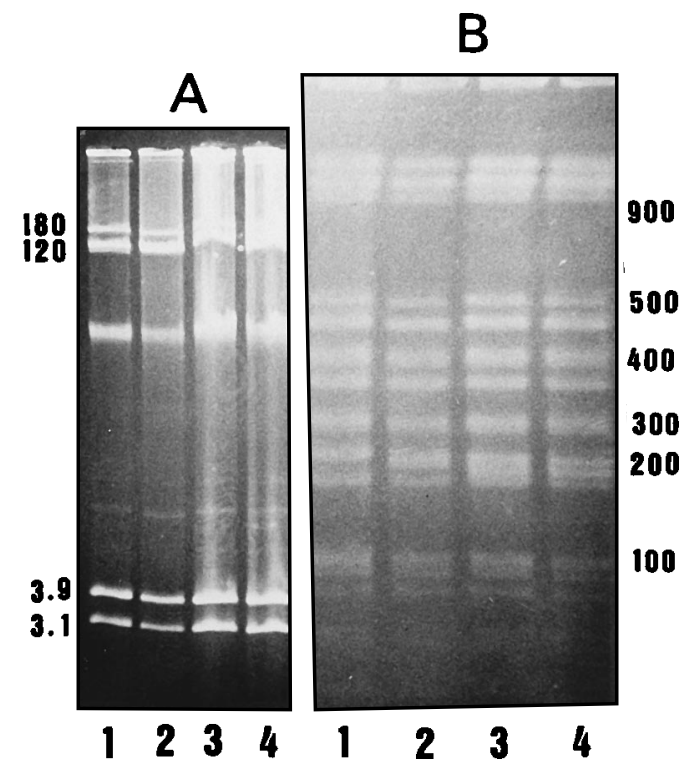

FIG. 2. Plasmid (A) and PFGE (B) patterns of $K$. pneumoniae LB1 (lanes 1), LB2 (lanes 2), LB3 (lanes 3), and LB4 (lanes 4). Molecular sizes (in kilobases) are indicated in the margins.

tained only the 35-kDa OMP from isolate LB1, whereas peaks II and III did not contain detectable proteins. Further analysis by SDS-PAGE and silver staining for LPS (Fig. 4C) demonstrated that the $35-\mathrm{kDa}$ OMP isolated in peak I was free of LPS detectable by this sensitive type of analysis, whereas peaks II and III contained, respectively, high-molecular-mass LPS (and some low-molecular-mass LPS) and low-molecular-mass LPS.

The results described above strongly suggested a porin nature for this protein. This was confirmed by the results shown in Fig. 5. When isolate LB4 was transformed with plasmid pSHA2, carrying the complete gene coding for the OmpK36 porin, an additional OMP, with an $M_{\mathrm{r}}$ coinciding with that of OmpK36, was observed in its outer membrane (Fig. 5A), while, as expected, LB4 transformed with a plasmid containing a truncated ompK36 gene did not show any OMP of 35 to 36 $\mathrm{kDa}$. Western blot analysis with antiserum against the OmpK36 porin (Fig. 5B) confirmed that the $35-\mathrm{kDa}$ OMP from LB1 and the 36-kDa OMP expressed by LB4 transformed with the ompK36-carrying plasmid are recognized by the anti-porin serum. Minor nonspecific reactions could be seen in some cases

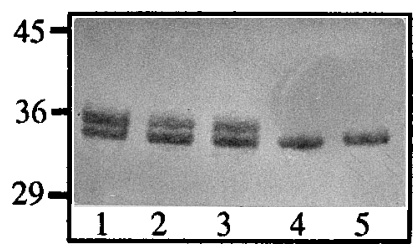

FIG. 3. SDS-PAGE analysis of OMP from isolates LB1 (lane 1), LB2 (lane 2), LB3 (lane 3), LB4 (lane 4), and LB2-FOX64, a cefoxitin-resistant laboratory mutant derived from $K$. pneumoniae LB2 (lane 5). Sizes of molecular mass markers (in kilodaltons) are indicated on the left. Only the relevant part of the gel is shown.

(Fig. 5B, lanes 1 and 3 but not lane 4) with the 32-kDa OMP common to LB1, LB2, LB3, and LB4. However, the specificity of the detection is shown by the absence of recognition of the OmpK35 porin of strain C3 (Fig. 5, lanes 6) by the antiOmpK36 serum.

Finally, the porin nature of the $35-\mathrm{kDa}$ OMP expressed by isolates LB1, LB2, and LB3 can be deduced from the fact that isolate LB4 transformed with plasmid pSHA2 carrying the porin-coding gene ompK36 reverted in its antimicrobial susceptibility levels to those of isolate LB2 (Table 1).

\section{DISCUSSION}

There are several reports on the association of loss of porins and increased resistance to antimicrobial agents, mainly in $E$. coli and Salmonella typhimurium $(17,25,35)$. For other gramnegative bacteria the association of loss of OMP in the range of 30 to $50 \mathrm{kDa}$ and resistance to antimicrobial agents has been considered evidence of the role of these OMP as porins. However, in many cases the exact nature of the protein has not been established, and the assumption that the involved proteins were porins were largely based on their molecular masses. This has been the case for several reports concerning $K$. pneumoniae $(31,36,47)$.

Two nonspecific porin proteins have been described for $K$. pneumoniae, named variously $37 \mathrm{kD}$ and $39-40 \mathrm{kD}(19,41)$ or OmpK36 and OmpK35 $(2,13)$. The three initial clinical isolates of our series, LB1, LB2, and LB3, lacked one of these porins, but the presence of the $35-\mathrm{kDa}$ one prevented the high level of resistance to expanded-spectrum cephalosporins and cefoxitin observed in isolate LB4. In isolate LB4 the absence of the $35-\mathrm{kDa}$ porin, together with the expression of the SHV-5 enzyme, account for the high level of resistance observed. The

TABLE 1. MICS of antimicrobial agents against evaluated strains

\begin{tabular}{|c|c|c|c|c|c|c|c|c|c|c|c|c|c|c|c|}
\hline \multirow{2}{*}{ Strain } & \multicolumn{15}{|c|}{ MIC $(\mu \mathrm{g} / \mathrm{ml})$ against $^{a}$ : } \\
\hline & AMP & $\mathrm{CR}$ & FOX & CAZ & CAZ-CAC & $\mathrm{AZT}$ & CTX & CTX-CAC & PIM & IMP & $\mathrm{AK}$ & G & CIP & $\mathrm{C}$ & $\overline{\mathrm{TE}}$ \\
\hline LB1 & $>256$ & 2 & 4 & 1 & $\leq 0.125$ & 0.125 & 0.06 & $\leq 0.125$ & 0.03 & 0.25 & 0.5 & 16 & 0.5 & $>128$ & 64 \\
\hline LB2 & $>256$ & 128 & 4 & $>256$ & $\leq 0.125$ & $>256$ & 2 & $\leq 0.125$ & 0.5 & 0.25 & 32 & 32 & 0.5 & $>128$ & 64 \\
\hline LB3 & $>256$ & 256 & 4 & $>256$ & $\leq 0.125$ & $>256$ & 4 & $\leq 0.125$ & 1 & 0.25 & 32 & 1 & 0.5 & 4 & 64 \\
\hline LB4 & $>256$ & $>256$ & 128 & $>256$ & 8 & $>256$ & 64 & 2 & $>8$ & 0.25 & 32 & 32 & 1 & $>128$ & 64 \\
\hline $\mathrm{KT}^{2} 005^{b}$ & $\mathrm{ND}^{c}$ & ND & 4 & $>256$ & $\leq 0.125$ & ND & 2 & $\leq 0.125$ & ND & ND & 8 & 4 & 0.5 & $>128$ & 64 \\
\hline KT5007 ${ }^{d}$ & ND & ND & 128 & $>256$ & 4 & ND & 64 & 1 & ND & ND & 8 & 16 & 1 & $>128$ & 64 \\
\hline
\end{tabular}

${ }^{a}$ AMP, ampicillin; CR, cephalothin; FOX, cefoxitin, CAZ, ceftazidime; CAZ-CAC, ceftazidime plus $2 \mu \mathrm{g}$ of clavulanic acid per ml; AZT, aztreonam; CTX, cefotaxime; CTX-CAC, cefotaxime plus $2 \mu \mathrm{g}$ of clavulanic acid per ml; PIM, cefepime; IMP, imipenem; AK, amikacin, G, gentamicin; CIP, ciprofloxacin; C, chloramphenicol; TE, tetracycline.

${ }^{b}$ LB4(pSHA2); expresses OmpK36 porin.

${ }^{c} \mathrm{ND}$, not determined.

${ }^{d}$ LB4(pSHA4); porin deficient. 


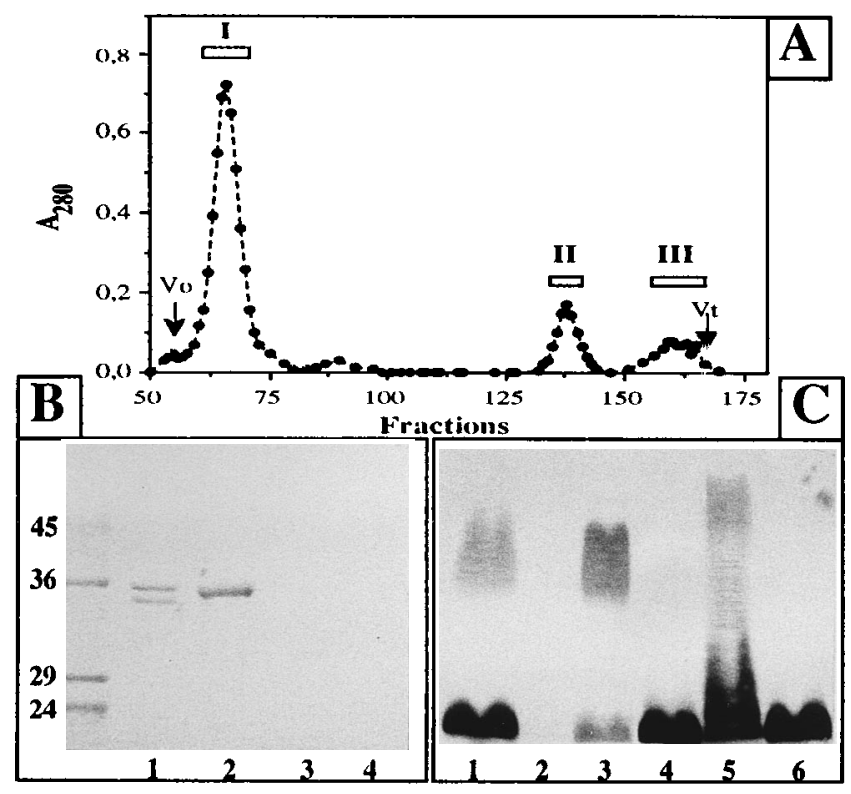

FIG. 4. Isolation of the $35-\mathrm{kDa}$ OMP from isolate LB1. (A) Gel permeation chromatography on Sephacryl S200 of cell envelopes subjected to a porin isolation method. (B) SDS-PAGE of relevant fractions. Lanes: 1, OMP from $K$. pneumoniae LB1; 2, peak I; 3, peak II; 4, peak III. (C). Fractions analyzed for LPS content. Lanes: 1, LPS from $K$. pneumoniae LB1; 2, peak I; 3, peak II; 4, peak III; 5, smooth LPS from an $S$. typhimurium strain; 6, rough LPS from an $S$. typhimurium strain. Sizes of molecular mass markers (in kilodaltons) are indicated on the left of panel B.

relationship between the porin deficiency and the resistance phenotypes is demonstrated in strain KT5005: cloning and expression of the ompK36 porin-coding gene in K. pneumoniae LB4 reverted the susceptibility of this strain to that of isolate LB2 (Table 1). Inactivation of cefoxitin by our clinical isolates was not detected. The resistance to this antimicrobial agent could not be reverted in vitro or transferred to $E$. coli by conjugation, therefore suggesting that isolate LB4 has a nonreversible chromosomal mutation. The resistance of LB4 and mutants resistant to high cefoxitin concentrations (MICs $>64 \mu \mathrm{g} / \mathrm{ml}$ ) is associated with the loss of the $35-\mathrm{kDa}$ porin.

Treatment of infections caused by $K$. pneumoniae strains producing ESBL is difficult not only because of their resistance to expanded-spectrum cephalosporins but also because of their resistance to other antimicrobial agents encoded by the same or different plasmids. Among $\beta$-lactams, cephamycin and imipenem seem to be reasonable alternatives, since preliminary data show that $\beta$-lactamases degrading cefoxitin and related drugs are uncommon, and resistance to imipenem in K. pneumoniae has not yet been described.

The results from this study show that cefoxitin may not be adequate for treatment of $K$. pneumoniae strains producing ESBL: mutants selected in vivo that lack porins (LB4, for instance) are resistant to cefoxitin, and cefoxitin-resistant, porin-deficient mutants are easily selected in vitro and show decreased susceptibilities to other $\beta$-lactams, including expanded-spectrum cephalosporins. According to National Committee for Clinical Laboratory Standards breakpoints for resistance, isolates LB2 and LB3 could be considered susceptible to cefotaxime. Although this is debatable, if the breakpoints proposed by some committees are considered $(4,26,42)$, the loss of the $35-\mathrm{kDa}$ porin makes $K$. pneumoniae LB4 a strain resistant to cefotaxime and other $\beta$-lactam antibiotics. The combination of cefotaxime with clavulanic acid (inhibiting SHV-5) determines a 32-fold decrease in the MIC of cefotaxime against $K$. pneumoniae LB4, but this combination is not commercially available and, in spite of this decrease, the MIC obtained is probably high enough to preclude its use. It could be interesting to study the activities of new combinations of expanded-spectrum cephalosporins with $\beta$-lactamase inhibitors to evaluate their possible clinical applications against $K$. pneumoniae strains producing ESBL. Interestingly, imipenem uniformly maintained its activity against these porinless mutants. This can be attributed to the small size of the molecule and its zwitterionic configuration, which facilitate its diffusion through the outer membrane. From these data, we suggest the use of imipenem if a $\beta$-lactam is considered for the treatment of infections caused by $K$. pneumoniae strains producing ESBL, and we suggest that the usefulness of other carbapenems be explored.

The susceptibility of isolate LB3 to both gentamicin and chloramphenicol was unexpected, as it contains the (apparently) same plasmid of $180 \mathrm{~kb}$ observed in isolates LB1, LB2, and LB4, which are resistant to both agents. New experiments have been planned to understand the different susceptibility pattern of isolate LB3.

This study was initially designed to study the mechanisms of resistance to $\beta$-lactam antibiotics. However, we have also noted that LB4 is slightly (but consistently) more resistant to ciprofloxacin than isolates LB1, LB2, and LB3 and mutant KT5005. This suggests that the loss of porins contributes to resistance to quinolones in $K$. pneumoniae. A two- to fourfold increase in MICs of hydrophilic quinolones has been similarly observed in E. coli mutants lacking OmpF. For our isolates, we still do not know if the diminished activity of ciprofloxacin is related to mutations in gyrase-coding genes or if it is due to a pleiotropic effect of the loss of the $35-\mathrm{kDa}$ porin. Also, the detection of an operon hybridizing with a mar-specific DNA probe (7) suggests that mar mutants may (or probably) exist in $K$. pneumoniae. New experiments are in progress to evaluate these mechanisms.

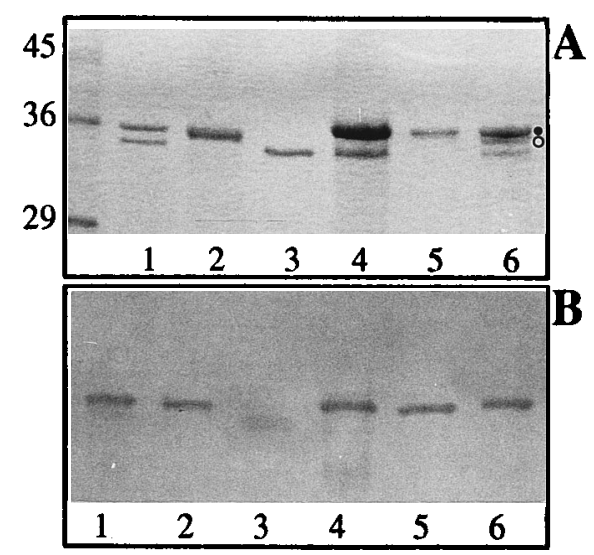

FIG. 5. SDS-PAGE (A) and Western blot with anti-OmpK36 serum (B) of OMP from $K$. pneumoniae LB1 (lanes 1), the $35-\mathrm{kDa}$ OMP purified from $K$. pneumoniae LB1 (lanes 2), OMP from $K$. pneumoniae LB4 carrying the truncated ompK36 gene of plasmid pSHA4 (lanes 3 ), OMP from $K$. pneumoniae LB4 carrying the ompK36-coding plasmid pSHA2 (lanes 4), purified OmpK36 porin (lanes 5); and OMP from K. pneumoniae C3 expressing porins OmpK36 (solid dot) and OmpK35 (empty dot) (lanes 6). Sizes of molecular mass markers (in kilodaltons) are indicated on the left of panel A. Only the relevant parts of the gel and Western blot are shown. 


\section{ACKNOWLEDGMENTS}

We thank F. Tenover (Centers for Disease Control, Atlanta, Ga.) for performing pulsed-field electrophoresis analysis, Víctor de Lorenzo (Centro de Investigaciones Biológicas, Madrid, Spain) for plasmid pDT1558, and Francisco Rodríguez-Quiñones (Centro Nacional de Biotecnología, Madrid, Spain) for plasmids pFR165 and pFR167.

This work was supported by grants PB91-0233-CO2 and PB93-0423 from Comisión Interministerial de Ciencia y Tecnología (CICYT) of the Government of Spain to V.J.B. and J.M.T. S.H.-A. and S.A. were supported by predoctoral fellowships from CICYT. The stay of L.M.-M. at Massachusetts General Hospital was supported by Sociedad Española de Enfermedades Infecciosas y Microbiología Clínica.

\section{REFERENCES}

1. Albertí, S., G. Marqués, C. Camprubí, S. Merino, J. M. Tomás, F. Vivanco, and V. J. Benedí. 1993. C1q binding and activation of the complement classical pathway by Klebsiella pneumoniae outer membrane proteins. Infect. Immun. 61:852-860.

2. Albertí, S., F. Rodríguez-Quiñones, T. Schirmer, G. Rummel, J. M. Tomás, J. P. Rosenbusch, and V. J. Benedí. 1995. A porin from Klebsiella pneumoniae: sequence homology, three-dimensional structure, and complement binding. Infect. Immun. 63:903-910.

3. Blake, M. S., K. H. Johnston, G. J. Russell-Jones, and E. C. Gotschlich. 1984. A rapid, sensitive method for detection of alkaline phosphatase-conjugated anti-antibody on Western blots. Anal. Biochem. 136:175-179.

4. British Society for Antimicrobial Chemotherapy. 1991. A guide to sensitivity testing. J. Antimicrob. Chemother. 27(Suppl. D):1-47.

5. Carpenter, J. L. 1990. Klebsiella pulmonary infections: occurrence at one medical center and review. Rev. Infect. Dis. 12:672-682.

6. Coetzee, J. N., N. Datta, and R. W. Hedges. 1972. R factors from Proteus rettgeri. J. Gen. Microbiol. 75:543-552.

7. Cohen, S. P., W. Yan, and S. B. Levy. 1993. A multidrug resistance regulatory chromosomal locus is widespread among enteric bacteria. J. Infect. Dis. 168: 484-488.

8. Dhaese, P., H. De Greve, H. Decraemer, J. Schell, and M. Van Montagu. 1979. Rapid mapping of transposon insertion and deletion mutations in the large Ti-plasmids of Agrobacterium tumefaciens. Nucleic Acids Res. 7:18371849.

9. Domenico, P., J. L. Marx, P. E. Schoch, and B. A. Cunha. 1992. Rapid plasmid isolation from mucoid gram-negative bacteria. J. Clin. Microbiol. 30: 2859-2863.

10. Filip, C., G. Fletcher, J. L. Wulf, and C. F. Earhart. 1973. Solubilization of the cytoplasmic membrane of Escherichia coli by the ionic detergent sodiumlauryl sarcosinate. J. Bacteriol. 115:717-722.

11. Gutmann, L., R. Williamson, N. Moreau, M.-D. Kitzis, J. F. Acar, and F. W. Goldstein. 1985. Cross-resistance to nalidixic acid, trimethoprim, and chloramphenicol associated with alterations in outer membrane proteins of Klebsiella, Enterobacter, and Serratia. J. Infect. Dis. 151:502-7.

12. Helander, I. M. 1985. Isolation and electrophoretic analysis of bacterial lipopolysaccharides, p. 263-274. In T. K. Korhonen, E. A. Dawes, and P. H. Mäkelä (ed.), Enterobacterial surface antigens. Methods for molecular characterisation. Elsevier, Amsterdam.

13. Hernández-Allés, S., S. Albertí, X. Rubires, S. Merino, J. M. Tomás, and V. J. Benedí. Isolation of FC3-11, a bacteriophage specific for the Klebsiella pneumoniae porin OmpK36, and its use for the isolation of porin-deficient mutants. Can. J. Microbiol., in press.

14. Herrero, M., V. de Lorenzo, and K. H. Timmis. 1990. Transposon vectors containing non-antibiotic resistance selection markers for cloning and stable chromosomal insertion of foreign genes in gram-negative bacteria. J. Bacteriol. 172:6557-6567.

15. Jacoby, G. A. 1994. Genetics of extended-spectrum beta-lactamases. Eur. J. Clin. Microbiol. Infect. Dis. 13(Suppl. 1):2-11.

16. Jacoby, G. A., and A. A. Medeiros. 1991. More extended-spectrum $\beta$-lactamases. Antimicrob. Agents Chemother. 35:1697-1704.

17. Jaffé, A., Y. A. Chabbert, and E. Delort. 1983. Selection and characterization of $\beta$-lactam-resistant Escherichia coli K-12 mutants. Antimicrob. Agents Chemother. 23:622-625.

18. Jeanteur, D., J. H. Lakey, and F. Pattus. 1994. The porin superfamily: diversity and common features, p. 363-380. In J.-M. Ghuysen and R. Hakenbeck (ed.), Bacterial cell wall. Elsevier, Amsterdam.

19. Kaneko, M., A. Yamaguchi, and T. Sawai. 1984. Purification and characterization of two kinds of porins from the Enterobacter cloacae outer membrane. J. Bacteriol. 158:1179-1181.

20. Korwick, J. A., C. S. Bryan, B. Farber, T. R. Bearn, L. Schaufeld, R. R. Muder, D. Weinbaum, R. Lumish, D. N. Gerding, M. M. Wagener, and V. L. Yu. 1991. Prospective observational study of Klebsiella bacteremia in 230 patients: outcome for antibiotic combinations versus monotherapy. Antimicrob. Agents Chemother. 36:2639-2644.

21. Laemmli, U. K. 1970. Cleavage of structural proteins during the assembly of the head of bacteriophage T4. Nature (London) 277:680-685.

22. Martínez-Martínez, L., S. Hernández-Alléz, V. J. Benedí, and G. A. Jacoby. 1994. Relationship of a $35 \mathrm{kDa}$ porin and increased resistance to cefoxitin and third generation cephalosporins in Klebsiella pneumoniae (KP), abstr. C67, p. 78. In Abstracts of the 34th Interscience Conference on Antimicrobial Agents and Chemotherapy. American Society for Microbiology, Washington, D.C.

23. Maslow, J. N., A. M. Slutsky, and R. D. Arbeit. 1993. Application of pulsefield gel electrophoresis to molecular epidemiology, p. 563-572. In D. H Persin, T. F. Smith, F. C. Tenover, and T. J. White (ed.), Diagnostic molecular microbiology. American Society for Microbiology, Washington, D.C.

24. Matthew, M., A. M. Harris, M. J. Marshall, and G. W. Ross. 1975. The use of analytical isoelectric focusing for detection and identification of $\beta$-lactamases. J. Gen. Microbiol. 88:169-178.

25. Medeiros, A. A., T. F. O'Brien, E. Y. Rosenberg, and H. Nikaido. 1987. Loss of OmpC porin in a strain of Salmonella typhimurium causes increased resistance to cephalosporins during therapy. J. Infect. Dis. 156:751-757.

26. Mensura-II. 1992. Reunión de la mesa española de normalización de la sensibilidad y resistencia a antibióticos. Rev. Esp. Quimioter. 5:155-158.

27. Meyer, K. S., C. Urban, J. A. Eagan, B. J. Berger, and J. J. Rahal. 1993 Nosocomial outbreak of Klebsiella infection resistant to late-generation cephalosporins. Ann. Intern. Med. 119:353-358.

28. Nikaido, H. 1983. Proteins froming large channels from bacterial and mitocondrial outer membranes: porins and phage Lambda receptor proteins. Methods Enzymol. 97:85-100.

29. Nikaido, H. 1989. Outer membrane barrier as a mechanism of antimicrobial resistance. Antimicrob. Agents Chemother. 33:1831-1836.

30. Nurminen, M. 1978. A mild procedure to isolate the $34 \mathrm{~K}, 35 \mathrm{~K}$ and $36 \mathrm{~K}$ porins of the outer membrane of Salmonella typhimurium. FEMS Microbiol. Lett. 3:331-334.

31. Pangon, B., C. Bizet, A. Buré, F. Pichon, A. Philippon, B. Regnier, and L. Gutman. 1989. In vivo selection of a cephamycin-resistant, porin deficient mutant of Klebsiella pneumoniae producing a TEM-3 Beta-Lactamase. J. Infect. Dis. 159:1005-1006.

32. Papanicolau, G. A., A. A. Medeiros, and G. A. Jacoby. 1990. Novel plasmidmediated $\beta$-lactamase (MIR-1) conferring resistance to oxyimino- and $\alpha$-methoxy $\beta$-lactams in clinical isolates of Klebsiella pneumoniae. Antimicrob. Agents Chemother. 34:2200-2209.

33. Philippon, A., R. Labia, and G. A. Jacoby. 1989. Extended-spectrum $\beta$-lactamases. Antimicrob. Agents Chemother. 33:1131-1136.

34. Philippon, A. M., G. C. Paul, and G. A. Jacoby. 1983. Properties of PSE-2 $\beta$-lactamase and genetic basis for its production in Pseudomonas aeruginosa. Antimicrob. Agents Chemother. 24:362-369.

35. Reguera, J. A., F. Baquero, J. C. Pérez-Díaz, and J. L. Martínez. 1991. Factors determining resistance to $\beta$-lactams combined with $\beta$-lactamase inhibitors in Escherichia coli. J. Antimicrob. Chemother. 27:569-575.

36. Rice, L. B., L. L. Carias, L. Etter, and D. M. Shlaes. 1983. Resistance to cefoperazone-sulbactam in Klebsiella pneumoniae: evidence for enhanced resistance resulting from the coexistence of two different resistance mechanisms. Antimicrob. Agents Chemother. 37:1061-1064.

37. Rice, L. B., S.H. Willey, A. Papanicolau, A. A. Medeiros, G. M. Eliopoulos, R. C. Moellering, and G. A. Jacoby. 1990. Outbreak of ceftazidime resistance caused by extended-spectrum $\beta$-lactamases at a Massachusetts chronic-care facility. Antimicrob. Agents Chemother. 34:2193-2199.

38. Sadowski, P. L., B. C. Peterson, D. N. Gerding, P. P. Cleary. 1978. Physica characterization of $10 \mathrm{R}$ plasmids obtained from an outbreak of nosocomia Klebsiella pneumoniae infection. Antimicrob. Agents Chemother. 15:616624

39. Sambrook, J., E. F. Fritsch, and T. Maniatis. 1989. Molecular cloning: a laboratory manual, 2nd ed. Cold Spring Harbor Laboratory Press, Cold Spring Harbor, N.Y.

40. Sanders, C. C., W. E. Sanders, R. V. Goering, and V. Werner. 1984. Selection of multiple antibiotic resistance by quinolones, beta-lactams, and aminoglycosides with special reference to cross-resistance between unrelated drug classes. Antimicrob. Agents Chemother. 26:797-801.

41. Sawai, T., S. Hirano, and A. Yamaguchi. 1987. Repression of porin synthesis by salicylate in Escherichia coli, Klebsiella pneumoniae and Serratia marcescens. FEMS Microbiol. Lett. 40:233-237.

42. Soussy, C. J., R. Cluzel, P. Courvalin, and the Comité de l-Antibiogramme de la Societé Francaise de Microbiologie. 1994. Definition and determination of in vitro antibiotic susceptibility breakpoints for bacteria in France. Eur. J. Clin. Microbiol. Infect. Dis. 13:238-246.

43. Szybalski, W. 1952. Microbial selection. I. Gradient plate technique for study of bacterial resistance. Science 116:46-48.

44. Tomás, J. M., V. J. Benedí, B. Ciurana, and J. Jofre. 1986. Role of capsule and $\mathrm{O}$ antigen in resistance of Klebsiella pneumoniae to serum bactericidal activity. Infect. Immun. 54:85-89.

45. Towbin, H., T. Staehelin, and J. Gordon. 1979. Electrophoretic transfer of proteins from polyacrylamide gels to nitrocellulose sheets: procedure and some applications. Proc. Natl. Acad. Sci. USA 76:4350-4354.

46. Tsai, C.-M., and C. E. Frasch. 1982. A sensitive silver stain for detecting lipopolysaccharides in polyacrylamide gels. Anal. Biochem. 119:115-119. 
47. van de Klundert, J. A. M., M. H. van Gestel, G. Meerdink, and S. de Marie. 1988. Emergence of bacterial resistance to cefamandole in vivo due to outer membrane protein deficiency. Eur. J. Clin. Microbiol. Infect. Dis. 7:776-777.

48. van der Ley, P., A. Bekkers, J. van Meersbergen, and J. A. Tommassen. 1987.

Comparative study on the phoE genes of three enterobacterial species. Eur. J. Biochem. 164:469-475.

49. Walter, E. G., and D. E. Taylor. 1989. Comparison of tellurite resistance determinants from the IncP $\alpha$ plasmid $\mathrm{RP}_{4} \mathrm{Te}^{\mathrm{r}}$ and the IncHII plasmid PHH1508a. J. Bacteriol. 171:2160-2165.

50. Werts, C., A. Charbit, S. Bachellier, and M. Hofnung. 1992. DNA sequence of the lamB gene from K. pneumoniae. Mol. Gen. Genet. 233:372-378.

51. Westphal, O., and K. Jann. 1975. Bacterial lipopolysaccharides: extraction with phenol-water and further applications of the procedure. Methods Carbohydr. Chem. 5:83-91. 